\title{
Cranial diabetes insipidus in early infancy
}

\author{
H R JENKINS, I A HUGHES, AND O P GRAY \\ Department of Child Health, University of Wales College of Medicine, Cardiff
}

SUMMARY We report an infant who developed cranial diabetes insipidus after septicaemic shock. This condition should be considered in any child who suffers an acute collapse and it may be more common in the high risk neonate than has previously been recognised.

The aetiology of cranial diabetes insipidus in children has been well documented, ${ }^{1}$ but the incidence in early infancy is unknown. This report concerns an infant who developed permanent diabetes insipidus after a hypotensive episode associated with meningococcal septicaemia.

\section{Case report}

A boy, birth weight $2500 \mathrm{~g}$, was born at term after a normal pregnancy. There were no perinatal problems and a routine ultrasound examination of the head gave a normal result before discharge at 6 days. The infant remained well until 6 weeks of age when he was readmitted to hospital after a sudden collapse. He was hypotensive and shocked and had a respiratory arrest soon after admission. A lumbar puncture gave normal results but meningococcus type $\mathrm{C}$ was subsequently isolated from blood cultures. He was treated with ceftazidime to which the organism was sensitive but he remained unwell and had convulsions. A cranial ultrasound examination three days after admission gave a normal result. After a transient improvement he became lethargic and began to vomit. The cranial ultrasound examination now showed dilatation of the ventricles.

Fluid intake had been restricted during this period and the serum sodium concentration rose to 153 $\mathrm{mmol} / \mathrm{l}$. This increased further to $164 \mathrm{mmol} / \mathrm{l}$ despite an adequate fluid intake and it was noted that he was passing large volumes of urine in excess of 30 $\mathrm{ml} /$ hour with a specific gravity fixed at $1 \cdot 000$. Plasma and urine osmolalities were 337 and $66 \mathrm{mosmol} / \mathrm{l}$, respectively and a diagnosis of diabetes insipidus was made. When the infant was rehydrated intravenously his condition improved. A further lumbar puncture gave a normal result and a cranial scan on computed tomography showed dilated ventricles which, in the absence of an increase in head circumference, suggested cerebral atrophy. A limited investigation of anterior pituitary function showed normal thyroid function and a normal random growth hormone concentration $(23.7 \mathrm{mU} / \mathrm{l})$; however, plasma cortisol concentrations were persistently low (<40 nmol/l). He was successfully treated with hydrocortisone $10 \mathrm{mg}$ and intranasal desmopressin, 0.5-1 $\mu \mathrm{g} /$ day. Plasma and urine osmolality and serum sodium returned to normal after three days. He has poor head growth and at 10 months of age he has appreciable developmental delay.

\section{Discussion}

Diabetes insipidus is rarely recognised in the neonatal period even though intracranial events such as hypoxic-ischaemic encephalopathy and intraventricular haemorrhage occur commonly at this time. Cranial diabetes insipidus, either permanent or transient, has been reported after intraventricular haemorrhage, ${ }^{2} 3$ listeria sepsis with disseminated intravascular coagulopathy, ${ }^{4}$ and streptococcal meningitis. ${ }^{5}$ This is the first report of permanent cranial diabetes insipidus developing in an infant after a hypoxic and hypotensive episode related to meningococcal septicaemia without meningitis. In addition the absence of a stress related increase in cortisol concentrations suggested deficiency of adrenocorticotrophic hormone. Hypotensive shock had presumably caused a reduction in cerebral blood flow sufficient to adversely affect hypothalamicpituitary function. Similar aetiological factors have been invoked in isolated growth hormone deficiency. ${ }^{6}$

Fluid restriction is recommended whenever there is a risk of cerebral oedema formation. This treatment regimen, however, may obscure the signs of diabetes insipidus unless fluid balance and osmolality are carefully monitored. It is possible that a proportion of cases of 'idiopathic' cranial diabetes insipidus may be due to problems unrecognised during the newborn period. The condition, especially in a transient form, should be considered as an important sequela of collapse in sick infants and in neonates who suffer intraventricular haemorrhage or hypoxic-ischaemic encephalopathy.

\footnotetext{
References

${ }^{1}$ Greger NG, Kirkland RT, Clayton GW, et al. Central diabetes
} 
insipidus-22 years' experience. Am J Dis Child 1986;140: 551-4.

2 Adams JM, Kenny JD, Rudolph AJ. Central diabetes insipidus following intraventricular hemorrhage. J Pediatr 1976;88:292-4.

3 Yu VYH, Werther GA. Treatment of neonatal diabetes insipidus with desmopressin (DDAVP). Aust Paediatr J 1980; 16::284-6.

${ }^{4}$ Fenton LJ, Kleinman LI. Transient diabetes insipidus in a newborn infant. J Pediatr 1974;85:79-81.

5 Pai KG, Rubin HN, Wedemeyer PP, et al. Hypothalamicpituitary dysfunction following group $\mathrm{B}$ beta hemolytic streptococcal meningitis in a neonate. J Pediatr 1976;88:289-91.
${ }^{6}$ Craft WH, Underwood LE, Van Wyk JJ. High incidence of perinatal insult in children with idiopathic hypopituitarism. J Pediatr 1980;96:397-402.

Correspondence to Dr HR Jenkins, Department of Child Health, University of Wales College of Medicine, Heath Park, Cardiff CF4 $4 \mathrm{XN}$.

Accepted 27 November 1987

\title{
Atopic eczema and preterm birth
}

\author{
T J DAVID AND C I EWING
}

Department of Child Health, University of Manchester

SUMMARY In a group of 443 children with atopic eczema there was a significant lack of subjects born before 37 weeks' gestation. It is possible that preterm birth reduces the chances of the subsequent development of severe atopic disease.

It has been suggested that antigen exposure in the first weeks of life may sensitise the immunologically immature infant, triggering the development of atopic disease. ${ }^{1}$ If this were true one might expect an increased incidence of atopic disease in subjects born preterm, and this has been examined in a group of children with atopic eczema.

\section{Subjects and methods}

Between January 1982 and August 1987, 445 children with atopic eczema were referred to the department of child health. Eighty were referred by paediatricians, 32 by dermatologists, 312 by general practitioners and 21 by other doctors. The gestational age at birth was recorded as part of a full history taken from the parents at the first visit. Three hundred and ninety two patients $(88 \%)$ were from the North West Region; 30 (7\%) were from the local health district of North Manchester. The rest came from other parts of England and Wales. The median year of birth of the patients was 1981/2. Information about the gestational age of children still alive who were born in 1981 and 1982, to women currently living in the North West Region, was obtained from the North West Regional Health Authority. In these two years there were 87606 children born who are still alive, and information about gestational age was available in $81038(93 \%)$.

\section{Results}

Information about gestation was unavailable in two patients who were adopted. There were three twins. The gestational age was reported to be 37 weeks or over in $428(97 \%)$ and less than 37 weeks in $15(3 \%)$. The gestational ages of the latter group were 36 weeks in seven children, 35 weeks in four, 34 weeks in three, and 29 weeks in one. The mean (SD) age of onset of the eczema was $6.8(11.2)$ months. The social class distribution of the parents of the patients was I and II, $169(38 \%)$; III, $170(38 \%)$; and IV and V, $106(24 \%)$. Fourteen patients were illegitimate, and the fathers of $38(9 \%)$ were unemployed. Of the 81038 children whose mothers were currently living in the region and where gestational age was known, $4803(6 \%)$ were less than 37 weeks' gestation and $76235(94 \%)$ were of 37 weeks' gestation or more. The distribution of gestation of the patients was significantly different from that of these 'control' children, $\chi^{2}=4 \cdot 66, p=0 \cdot 03$.

\section{Discussion}

In a group of 443 children with atopic eczema, there was an unexpected and significant lack of subjects born preterm. As in any hospital based study the patients were highly selected, mainly by severity and poor response to topical treatment, but also by social class. A further drawback is that information about gestation was obtained from parents rather than from maternity records. Nevertheless, although parents may have overestimated or underestimated gestational age these errors should cancel each other out, and there is no reason to suspect that parents of atopic children are especially prone to overestimate 